$$
(A+B) C \leqq A C+B C,
$$

and our theorem is proved.

We can also prove the following:

3.2. Corollary. A necessary and sufficient condition that

$$
(A+B) C=A C+B C
$$

for positive $A, B$, and $C$ is that either $C=1$, or $1<C<\omega$ and $\alpha_{0} \leqq \beta_{0}$, or $\omega \leqq C$ and $\alpha_{0}+\gamma_{0}<\beta_{0}+\gamma_{0}$.

This corollary follows quite easily from the reasoning found in the preceding section.

Cornell University

\title{
THE DECOMPOSITION THEOREM FOR ABELIAN GROUPS ${ }^{1}$
}

\section{JOEL BRENNER}

Let $G$ be an abelian group such that $p^{k} g=0$ for all $g \in G, p$ prime, $k$ fixed. We prove $G$ has a basis, that is, a set of elements such that each $g \in G$ is uniquely expressible as a linear combination of elements of the set. ${ }^{2}$

THEOREM. There exists an ascending chain of sets $B_{i}, 0 \leqq i \leqq k$, of elements of $G$ with the properties:

(i) Every element in $B_{i}$ is of order greater than $p^{k-i}$.

(ii) The elements in $B_{i}$ are completely linearly independent.

(iii) If the order of the element $g$ in $G$ is greater than $p^{k-i}$, then there exists a (unique) linear combination $z$ of elements of $B_{i}$ such that the order of $g-z$ is at most $p^{k-i}$.

Since we may choose as $B_{0}$ the vacuous set, we may assume that the sets $B_{0}, \cdots, B_{s}$ have already been constructed in such a way as to meet the requirements (i) to (iii). In order to construct $B_{s+1}$ we adjoin to $B_{s}$ any greatest subset $C$ of $G$ with the following properties.

(a) All the elements in $C$ are of order $p^{k-s}$.

(b) The join $B_{s+1}$ of the sets $B_{s}$ and $C$ is an independent set.

1 Presented to the Society, April 6, 1940.

${ }^{2}$ Unique in that the number of nonzero terms in an expression for $g$ is unique and only the arrangement but not the respective values of the nonzero terms may differ in two expressions for $g$. 
The set $B_{s+1}$ satisfies (i) and (ii). In order to prove (iii) let $h$ be any element in $G$ whose order is at least $p^{k-s}$. If firstly the order of $h$ is exactly $p^{k-s}$, then it follows from the conditions (a) and (b) that there exists an integer $m$ and a linear combination $y=\sum a_{j} y_{j}$ of elements in $B_{s+1}$ so that $0 \neq m h=y$. Assume without loss of generality that $m$ is a power of $p<p^{k-s}$. Then $\left(p^{k-s} / m\right) m h=0=\sum a_{j}\left(p^{k-s} / m\right) y_{j}$. By (ii), $p^{k-s}$ divides $a_{j}\left(p^{k-s} / m\right)$, so that $a_{j} / m$ is an integer (all $j$ ). Therefore the order of $h-\sum\left(a_{j} / m\right) y_{j}$ is $m<p^{k-s}$. If secondly the order of $h$ is greater than $p^{k-s}$, then there exists a linear combination $z$ of elements in $B_{s}$ such that the order of $h-z$ is at most $p^{k-s}$, and thus (as above) $y=\sum c_{j} y_{j}$ can be found with $y_{j}$ in $B_{s+1}$ for which $h-z-y$ has order $<p^{k-s}$. We have shown ${ }^{3}$ that $B_{k}$ is a basis of $G$.

\section{UNIVERSITY OF CALIFoRNiA}

${ }^{3}$ The following contain proofs for finite groups: Andreas Speiser, Theorie der Gruppen von endlicher Ordnung, 3d edition, Berlin, Julius Springer, 1937, p. 46-49. R. Remak, Über die Zerlegung der kommutativen Gruppen in zyklische teilerfremde Faktoren, Journal für die reine und angewandte Mathematik, vol. 141. L. C. Mathewson, A simple proof of a theorem of Kronecher, ibid., vol. 161 (1929), p. 255. A. Korselt, ibid. N. Tschebotaroew, Bewies der Existenz einer Basis bei Abelschen Gruppen von endlicher Ordnung, Kasan University, Fizzico-Matematico Obchestvo Izviestiia, vol. 4 (1929-1930). Third line after $\delta$ ) in the proof read $\omega=\omega_{s} r$ for $\omega=\omega_{s}=r$. In the fourth paragraph following, read $\tilde{\omega}_{s}=q \omega_{s}+t, 0 \leqq t<\omega, A_{s} \cdots t \cdots$. The following contain proofs for infinite groups: H. Pruefer, Untersuchungen ueber die Zerlegbarkeit, Mathematische Zeitschrift, vol. 17 (1923), p. 53; R. Baer, Compositio Mathematica, vol. 1 (1934), p. 274. 